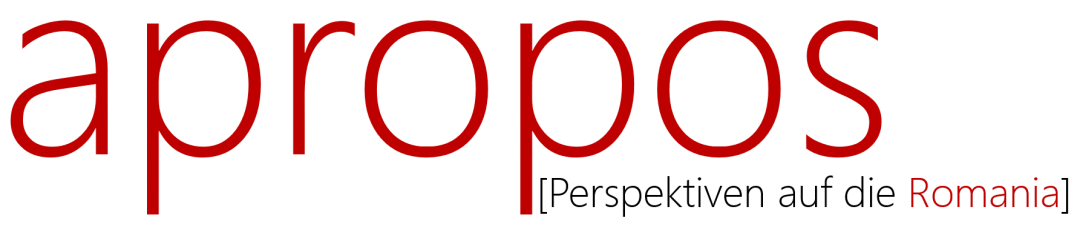

Sprache/Literatur/Kultur/Geschichte/Ideen/Politik/Gesellschaft

$1 \mid 2018$

Romanistik aktuell

"Halb Courteline, halb Ubu, halb Kafka"
Forschungen zur neueren französischen
Universitätsgeschichte nach 1968
Markus Bodler
apropos [Perspektiven auf die Romania]
Hamburg University Press
$\begin{aligned} & \text { 2018, } 1 \\ & \text { pp. } 145-160\end{aligned}$

ISSN: $2627-3446$

$\mathrm{DOl}$

https://doi.org/10.15460/apropos.1.1263

Zitierweise

Bodler, Markus. 2018. „Halb Courteline, halb Ubu, halb Kafka” Forschungen zur neueren französischen Universitätsgeschichte nach 1968“, apropos [Perspektiven auf die Romania] 1, 141-156. DOI: 10.15460/apropos.1.1263

Except where otherwise noted, this article is licensed under a Creative Commons Attribution 4.0 International license (CC BY 4.0)

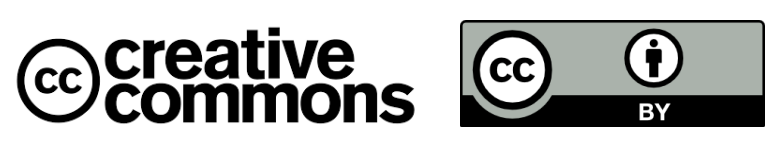




\title{
Markus Bodler
}

\section{„Halb Courteline, halb Ubu, halb Kafka“ Forschungen zur französischen Universitätsgeschichte nach dem Mai 1968}

\section{Sammelrezension}

\author{
Markus Bodler \\ ist Dozent im FUBiS-Programm der Freien \\ Universität Berlin \\ markus.bodler@fu-berlin.de
}

BOURILLON, Florence et al. (ed). 2016. De l'Université de Paris aux universités de l'île-de-France. Rennes: PUR.

DORMOY-RAJRAMANAN, Christelle. 2014. Sociogenèse d'une invention institutionnelle. Le Centre universitaire expérimental de Vincennes. (Thèse présentée pour obtenir le grade de Docteure, sous la direction du Prof. Bernard Pudal. Université de Paris Ouest-Nanterre la Défense, $<$ https:// bdr.parisnanterre.fr/theses/internet/2014PA100149.pdf>)

Mercier, Charles. 2015. Autonomie, autonomies. René Rémond et la politique universitaire aux lendemains de Mai 1968 (collection Histoire de la France aux XIXe et $X{ }^{e}$ siècles, 77). Paris: Publications de la Sorbonne.

Mercier, Charles. 2016. René Rémond et Nanterre. Les enfantements de 68 (1968-1976). Lormont: Le Bord de l'eau.

VALENCE, David \& Bruno Poucet (ed.). 2016. La loi Edgar Faure. Réformer I'université après 1968. Rennes: PUR.

\section{Keywords}

1968 - Universität - Edgar Faure - Reform - René Rémond

Die französische Universitätslandschaft sah einst aus wie der Schlosspark von Versailles, wohlgeordnet und in perfekter Harmonie. So gleichmäßig unterteilt war sie in Akademien mit je einem Rektor und je einer Universität, dass offenkundig überall Plan und Methode regierten. An der Basis standen zudem die immer gleichen Fakultäten, fünf an der Zahl: Medizin, Pharmazie, Geisteswissenschaften, Naturwissenschaften und Recht. Ganz oben schließlich thronte, als höchste staatliche Instanz und Schlussstein des Ganzen, das Pariser Erziehungsministerium.

Dann aber kam Edgar Faure, und mit der schönen Ordnung war es dahin. Dieser Mann, der von der Radikalen Partei, die ihn, wie er selbst sagte, vorübergehend verlassen hatte, zu den Gaullisten gewechselt war, übernahm nach dem 
Mai 1968 ein Jahr lang den Posten des Erziehungsministers. Sein Auftrag war es, die Universitäten grundlegend zu reformieren. Mit ihm wurde aus dem Schlosspark ein englischer Landschaftsgarten. Allein in der Pariser Region entstanden anstelle der einen Sorbonne 13 Universitäten, inzwischen sind es gar 17. Der Pariser Rektor, in seiner Akademie so etwas wie der Préfet des Erziehungsministeriums, musste sich seine Befugnisse als oberste Aufsichtsinstanz von nun an mit gewählten Universitätspräsidenten teilen. Und aus den fünf in Stein gemeißelten Fakultäten wurde eine unübersehbare Anzahl an Lehrund Forschungseinheiten, sogenannte Unités d'enseignement et de recherche (UER), deren fachliche Bezeichnungen obendrein stark variierten und überhaupt ganz uneinheitlich waren. Mit einem Wort, es hatte eine Revolution stattgefunden.

Um so verwunderlicher ist, dass die französische historische Forschung es lange versäumt hat, sich dieser Revolution anzunehmen. Außer in Überblicksdarstellungen, die dem Universitätsgesetz von Faure naturgemäß nur wenig Raum widmen können, wurde es bislang nicht genauer untersucht, weder in den Arbeiten zum Mai 68 noch in denen zum französischen Erziehungssystem. Überhaupt muss man sagen, dass die französischen Historiker die neuere Universitätsgeschichte lange Zeit recht stiefmütterlich behandelt haben. Mit Vorliebe bearbeitet wurden hingegen andere Themen, wie Schulgeschichte oder das Universitätssystem der frühen III. Republik. Die Gründe dafür werden immer noch eifrig diskutiert. Eine Vermutung ist, dass die vielen jungen Universitäten erst allmählich darauf aufmerksam wurden, wie wichtig die historischen Anfänge tatsächlich sind für ihre eigene, sich erst allmählich festigende Identität, zumal im Wettbewerb mit anderen Universitäten aus dem In- und Ausland. Entscheidender, zumindest aus praktischer Sicht, dürfte aber wahrscheinlich der Umstand sein, dass erst im Laufe der letzten zehn Jahre die historische Überlieferung der Universitäten mehr und mehr zugänglich wurde. Gab es noch 2006 in ganz Frankreich lediglich drei funktionierende Universitätsarchive, war es wenige Jahre später schon um ein Vielfaches mehr. Dank dieser archivalischen Kärrnerarbeit wanderten die Dokumente, inventarisiert und geordnet, aus den Kellern der Hochschulen in die Lesesäle des Nationalarchivs und der Departementsarchive, aber auch in die winzigen Büros der Universitätsarchive. In der Folge, so darf man wohl sagen, wurde ein wahres Füllhorn an neuen Quellen über die Historiker ausgeschüttet.

Unter diesen Umständen nimmt es nicht Wunder, wenn der erste Band zur neueren französischen Universitätsgeschichte, der hier vorgestellt werden soll, auf die Initiative einer Archivarin zurückgeht: Stéphanie Méchine, Leiterin des Archivs des Pariser Rektorats und in gewisser Weise Schnittstelle für alle Universitätsarchive in Paris und Umland. Sie hat ein Kolloquium angestoßen, das Anfang 2014 die Vertreter aller relevanten Fächer zusammenbrachte - von Stadtplanern und Architekten über Politikwissenschaftler und Historiker bis hin zu Bibliothekaren und Archivaren - und dessen Beiträge zwei Jahre später unter dem Titel De l'Université de Paris aux universités d'île-de-France veröffentlicht wurden. 
In der Einleitung dieses Sammelbandes gibt der Stadthistoriker Loïc Vadelorge den Ton für alle weiteren Arbeiten vor. Statt von einer Neugründung der Pariser Universität, in gütlicher, allseitiger Einigung, spricht er ganz bewusst von einem Erbfolgekrieg. In der Tat war eine der entscheidenden Fragen bei der Aufteilung der Sorbonne, welche der Nachfolgeuniversitäten denn nun was bekommen soll. So kam es zu heftigen Rivalitäten. Dabei ging es nicht nur um Vorlesungssäle, Seminarräume und Bibliotheken, also um das materielle Erbe; bedeutsam war darüber hinaus auch der symbolische Wert. Wer also sollte die Sorbonne weiter im Namen führen, wer das Universitätssiegel übernehmen und wer seine Verwaltung im alten Gebäude auf der Montagne Sainte-Geneviève unterbringen dürfen? Es war, möchte man sagen, wie der Streit um den Tempelberg in Jerusalem.

Von diesem Erbschaftsstreit erzählt auf souveräne Art und Weise Christophe Charle, der Altmeister einer an Bourdieu orientierten Sozialgeschichte. In seinem Beitrag erfährt man, dass es in den Jahren nach der Universitätsreform zu einer Art Wohngemeinschaft mehrerer Universitäten unter einem Dach kam. Dass dies zu Spannungen führte, wird niemanden überraschen. Charle zitiert insbesondere aus einem Bericht von 1973, den der Althistoriker Henri van Effenterre in seiner Eigenschaft als Beauftragter für die Raumvergabe an der Universität Paris I verfasst hat. Effenterre fällt darin ein bitteres, vernichtendes Urteil. So wurde seiner Meinung nach die Aufteilung der Sorbonne „mit dem Scharfsinn eines Provinznotars" vollzogen, "dessen Geschicklichkeit darin besteht, eine profitable Spannung zwischen den ineinander verflochtenen Erben aufrechtzuhalten". Und über die Politik der Raumvergabe an den Pariser Nachfolgeuniversitäten urteilt er launisch, aber wohl nicht weniger zutreffend: „Halb Courteline, halb Ubu, halb Kafka“. Oder mit anderen Worten: grotesk und absurd, willkürlich und voller Machtspiele und darüber hinaus auch noch bürokratisch und undurchschaubar.

Wer nun glaubt, dass es denjenigen Pariser Universitäten besser erging, die in eigens für sie errichtete Gebäude untergebracht wurden, sieht sich getäuscht. Ihre Geschichte wird gleich von mehreren Autoren erzählt, oft verbunden mit Fragestellungen aus der Stadtplanung: wie etwa urbane Räume neugestalten (beispielsweise den alten Pariser Weinmarkt an der Seine), wie neue Grundstücke erwerben (nicht selten vom französischen Militär) oder wie die universitäre Reform architektonisch umsetzen? Ein architekturgeschichtlicher Ausnahmefall war dabei die Reformuniversität Dauphine, das spätere Paris IX. Hier genügte es, den ehemaligen Sitz der NATO universitätsgerecht umzubauen und gemäß den neuen pädagogischen Vorgaben auszustatten; anstelle der üblichen großen Hörsäle entstanden so viele kleine Seminarräume. In den Neubauten hingegen wurde wegen des enormen Zeitdrucks fast durchweg auf Fertigbauweisen gesetzt. Dies trifft nicht nur auf das spätere Paris VIII zu, das im Park von Vincennes innerhalb weniger Wochen entstand, sondern gleichfalls auf die schon Mitte der 1960er Jahre erbauten Universitätsgebäude von Nanterre, aus dem die Universität Paris $X$ hervorging. Doch so sehr es als Leistung der Architekten zu werten ist, alle administrativen Hürden bewältigt und die 
Bauvorhaben in rasantem Tempo fertiggestellt zu haben, im Ergebnis blieb oft nur das, was die damalige Presse wie im Falle von Nanterre übereinstimmend als „freudlos" und "seelenlos" beschrieb oder, kurzum, als „architektonische Misere". Überdies sollte in späteren Jahren manch neue Universität die eigene Baugeschichte einholen. Nicht selten waren so schon bald nach der Fertigstellung umfangreiche Renovierungsarbeiten oder Asbestsanierungen erforderlich.

Die Reformen Edgar Faures hatten jedoch nicht nur zum Ziel, die aus allen Nähten platzende Sorbonne räumlich zu entlasten. Im Kern ging es um viel mehr. Eine der grundlegenden Ideen war, die Voraussetzungen für ein ungehindertes Zusammenspiel aller Disziplinen zu schaffen. Ganz besonders erwünscht war dabei die Zusammenarbeit der Geistes- und Naturwissenschaften. Dazu mussten aber erst einmal die engen Grenzen gesprengt werden, die von den überkommenen Fakultäten gesetzt wurden. Denn Kooperationen über Fächergrenzen hinweg sollten erleichtert werden, ebenso wie die Einführung neuer Disziplinen, etwa in den Wirtschafts- und Sozialwissenschaften. Aus diesem Grund beschloss Faure, gleich einem Urknall, erst einmal alle bisherigen fachlichen und überfachlichen Verbindungen aufzulösen, um sie von unten her wieder aufzubauen, nämlich mittels der bereits genannten UER, der Basiseinheit aller neu zu schaffender, möglichst pluridisziplinärer Universitäten. Dies war jedenfalls die Theorie. In der Praxis jedoch folgte auf die Scheidung in den meisten Fällen recht schnell die Wiederheirat mit den alten Partnern. Denn so wie vor allem Juristen und Naturwissenschaftler wollten auch die meisten anderen Professoren der Sorbonne weiterhin lieber unter sich bleiben.

Wie wichtig aber dieses pluridisziplinäre Prinzip dem Ministerium war - nicht nur Faure selbst, sondern auch seinen Nachfolgern - zeigte sich ganz besonders im Fall der Universität Paris VII. Deren Entstehungsgeschichte beschreibt Arnaud Desvignes, einer der jüngeren Spezialisten für Universitätsgeschichte. Aus seinem Beitrag geht hervor, wie diese Universität nur dank staatlichen Drucks und gegen den ausdrücklichen Willen der Professoren ins Leben gerufen wurde. In der Folge stand, weil politisch gewollt, der von den Naturwissenschaften dominierten Universität Paris VI eine Universität mit großer fachlicher Vielfalt gegenüber, eben Paris VII.

Allerdings war eine derartige Intervention von oben alles andere als üblich. Vielmehr bildeten sich die UER und die neuen Universitäten in der Regel durch freiwilligen Zusammenschluss. Die Professoren und Assistenten taten sich aus eigenem Antrieb zusammen, zumeist aufgrund von Wahlverwandtschaften. Dabei kam es nicht nur darauf an, dass man das gleiche Fach vertrat, sondern auch ob man wissenschaftlich und politisch auf einer Linie war oder, anders gesagt, sich für oder gegen die Ideen der 68er-Bewegung ausgesprochen hatte. Nicht unwesentlich waren auch Freundschaften, solche aus der Studienzeit etwa. Leider aber wirft der vorliegende Band auf diesen Prozess der Neugruppierung nur sehr wenig Licht. Im Dunkeln bleibt beispielsweise, wie genau sich Paris I den Ruf einer linken, Paris IV aber den einer rechten Universität erwarb. Ebenso zu kurz kommen darüber hinaus auch die Inhalte von 
Lehre und Forschung (und welcher Änderungen sie unterworfen sind) oder die Frage, wie genau sich an den Universitäten in der Zeit nach dem Mai 68 Wissenschaft und Politik neu verbanden. Freilich gehen solche Fragen auch weit über das eigentliche Ziel des Sammelbandes hinaus, zumal für ihre Beantwortung wohl auch ganz andere Arten von Quellen nötig wären als Berichte und Protokolle der Universitätsgremien jener Jahre.

Was nun aber die eigentlich politischen Fragen der Universitätsreform anbetrifft, greift man ohnehin besser zu einem anderen Werk: dem ebenfalls 2016 herausgegebenen Sammelband La loi Edgar Faure. Réformer l'université après 1968, der auf einem von der Fondation Charles de Gaulle im November 2011 organisierten Kolloquium beruht. Auch hier wurden neue Quellen ausgewertet, speziell die Archive von Mitarbeitern und Beratern, die im ElyséePalast für den Bereich Universitätspolitik zuständig waren. In der Summe wird dem Leser so nicht nur ein tiefer Einblick in die gaullistische Seele ermöglicht, sondern auch ein breites Panorama geboten, das von den parteieigenen Interesseverbänden über die Gruppe der Parlamentsabgeordneten bis hin zu den Premierministern und de Gaulle selbst reicht. Was man aus der Lektüre der einschlägigen Erinnerungen von Alain Peyrefitte und Jacques Foccart bereits halbwegs zu kennen glaubte, wird damit auf eine sehr solide Grundlage gestellt, zudem politisch eingeordnet und das weit über das direkte Umfeld de Gaulles hinaus.

Am Anfang des Bandes steht eine überraschende Beobachtung: wie unproblematisch und scheinbar spielend leicht es war, die Universitätsreform politisch durchzusetzen. Von Faure und seinem Team an Beratern in nur sechs Wochen erarbeitet, verabschiedete das Parlament das Gesetz im November 1968 im Schnelldurchlauf. Kein einziger Abgeordneter der Nationalversammlung stimmte dagegen, und nur die Kommunisten zusammen mit fünf Gaullisten enthielten sich der Stimme. Von außen gesehen gelang also alles außerordentlich schnell und verblüffend reibungslos. Intern aber hagelte es Kritik. Man war entsetzt, ein Aufschrei ging durch die gaullistische Bewegung.

Das eigentliche Thema ist denn auch die gaullistische Fundamentalopposition gegen die Reformen, so wie sie von Faure, dem parteifremden, von de Gaulle eingesetzten Feuerwehrmann, angestrebt wurden. Detailliert beschrieben wird dies insbesondere von Antoine Prost, der als anerkannte Autorität für die Geschichte des französischen Bildungssystems den zentralen Artikel verfasst hat, sowie von François Audigier, einem Gaullismus-Experten, der sich den Seelenschmerzen der Parlamentarier widmet. Dabei wird klar, dass die neuen Ideen überall in der gaullistischen Bewegung auf heftigen Widerstand stießen. Nicht nur der Premierminister und der Finanzminister gehörten zu den Gegnern, auch die übergroße Mehrheit der Abgeordneten, ja selbst Faures eigener Staatssekretär. Bezeichnenderweise soll als Reaktion auf eine erste Version des Gesetzestextes der Generalsekretär des Elysée-Palastes ausgerufen haben: "Quelle horreur!... Quelle horreur!" 
Allerdings ist es nun auch wirklich verblüffend, dass die Gaullisten, die in den Parlamentswahlen Ende Juni 1968 noch einen Erdrutschsieg gefeiert hatten, plötzlich eine politische Agenda verfolgten, die in fast allen Punkten ihren eigenen Präferenzen widersprach. Vor dem Mai 68 hatte man jedenfalls noch das Ziel ausgerufen, den Zugang zu den Universitäten einer stärkeren staatlichen Kontrolle zu unterziehen und insbesondere ein strenges Auswahlverfahren einzuführen, einschließlich verpflichtender Beratungen, mit deren Hilfe die Studenten zu den Fächern gelenkt werden sollten, die sie sinnvollerweise und mit guten Berufsaussichten studieren konnten, zum Nutzen nicht nur der Wirtschaft, sondern der gesamten Nation. Davon war nun keine Rede mehr. Stattdessen sah neben der Pluridisziplinarität das Rahmengesetz Faures zwei weitere Prinzipien vor, die in gaullistischen Kreisen höchst umstritten waren, nämlich eine gewisse universitäre Autonomie und eine Teilhabe vor allem der Studenten an den Entscheidungen ihrer Universität, die sogenannte Partizipation. Während man bei ersterem angesichts der vielen neuen verantwortlichen Instanzen ein heilloses Durcheinander in der Universitätspolitik befürchtete, im Grunde also Angst hatte vor einer IV. Republik an den Hochschulen, sah man bei letzterem die Gefahr der Politisierung und schreckte davor zurück, die Universitäten eventuell in die Hand linksradikaler Studenten geben zu müssen. Für die Gaullisten hatten die beiden Prinzipien also Mängel in Hülle und Fülle, in erster Linie aber vermissten sie schlicht die ordnende Hand des Staates. Kaum vorstellbar jedenfalls, dass es Wahlgewinner gibt, die über ihre eigene Gesetzgebung weniger glücklich waren als die Gaullisten von 1968. Da half es dann auch nicht mehr, dass die eigene Abgeordnetenmehrheit zumindest darin erfolgreich war, das Gewicht der Studenten in den universitären Entscheidungsgremien einigermaßen zu begrenzen.

Im Ergebnis bleiben allerdings zwei offene Fragen, die die Autoren des Sammelbandes auch zu beantworten versuchen. Zum einen: Warum nur gehorchten die Abgeordneten und stimmten von ganz wenigen Ausnahmen abgesehen für die Reform? Zum anderen: Warum änderte de Gaulle seine Politik, setzte Faure als Minister ein und stützte ihn, trotz manch eigener Zweifel, solange er selbst an der Macht war? Auf die erste Frage gibt François Audigier eine ganz und gar unzweideutige Antwort. Der Gaullismus, so seine Einschätzung, sei 1968 noch eine politische Familie gewesen, in der quasimilitärischer Gehorsam zum guten Ton gehörte. Damit erscheint jedoch die zweite Frage umso bedeutsamer. Auf sie versucht einer der Herausgeber, Bruno Poucet, eine plausible Antwort zu finden. In seinem Schlusswort schreibt er, dass es de Gaulle wie schon zuvor beim Algerienkrieg darum gegangen sei, Wunden zu heilen und die nationale Einheit wiederherzustellen, jetzt eben im universitären und studentischen Milieu, so fremd es inm, dem General, auch immer geblieben ist. Man könnte freilich hinzufügen, dass diese These noch einen anderen Punkt berührt, nämlich den der für de Gaulle so wichtigen Gewissheit über die eigene Legitimität. Sie wollte er mit den Neuwahlen im Juni 1968 wiedererlangen und, mehr noch, mit der Ankündigung eines Referendums. Nicht zuletzt aber fügen sich in diesen Kontext auch die beiden Stichworte, die 
de Gaulle seit Mai 1968 selbst als politische Losung ausgegeben hat und die in dem vorliegenden Band auch immer wieder fallen: Partizipation und Dezentralisierung. Somit sind universitäre Mitbestimmung und universitäre Autonomie, und zwar nicht nur in Paris, sondern in allen Regionen Frankreichs, Teile einer größeren Reform, die de Gaulle im Laufe eines Jahres zu Ende führen wollte; doch scheiterte er mit diesem Vorhaben im April 1969, als sein Referendum keine Mehrheit fand.

Dies waren nun, im gerafften Überblick, einige der Ergebnisse zweier wissenschaftlicher Kongresse, die dank zahlreicher, bis vor kurzem noch unausgewerteter Quellen einen weit tieferen Einblick in das Reformwerk Edgar Faures gestatten, als es bisher möglich war. Dabei ist es durchaus empfehlenswert, die Texte parallel zu lesen und sie als einander ergänzende Einheit zu betrachten, oder anders gesagt: als zwei Seiten einer Medaille.

An beiden Kolloquien nahmen im übrigen auch Forscher der jüngeren Generation teil, so wie der bereits genannte Arnaud Desvignes (Paris IV), darüber hinaus Charles Mercier (Paris I) und Christelle Dormoy-Rajramanan (Paris X). Alle drei sind Verfasser gewichtiger Dissertationen, sogenannter thèses de doctorat. Bislang jedoch wurden nur die Arbeiten der beiden Letztgenannten veröffentlicht; sie sollen im folgenden besprochen werden: die thèse von Mercier über den ersten Präsidenten der Universität Nanterre, publiziert in zwei Bänden, und die von Dormoy-Rajramanan über die Entstehungsgeschichte der Universität Vincennes, die im Internet als PDF-Datei frei verfügbar ist. Vom Thema, den Reformuniversitäten der 1968er Jahre, einmal abgesehen, haben die Forschungen der beiden Nachwuchswissenschaftler allerdings nur sehr wenig gemeinsam. Doch so verschieden, ja unvereinbar ihre wissenschaftlichen Ansätze auch sind: In beiden Fällen handelt es sich letztlich um gut recherchierte, quellengesättigte Arbeiten, die mit um die tausend Seiten auch eine beachtliche Länge haben.

Ursprünglich allerdings wollte Mercier eine umfassende Biographie über den Zeithistoriker René Rémond schreiben. Der Vernunft gehorchend, entschloss er sich dann aber, das Thema einzugrenzen, und konzentrierte sich auf die Jahre 1968 bis 1976, in denen Rémond nach anfänglichen Schwierigkeiten den Nachweis erbrachte, dass in ihm ein geborener Administrator und gewiefter Universitätspolitiker steckte. Der breiten Öffentlichkeit bekannt wurde Rémond jedoch nicht als Universitätspräsident, sondern als Fernsehkommentator, der an Wahlabenden, bewaffnet mit politischer Logik und einem schier unerschöpflichem Arsenal an historischen Bezügen, die Resultate analysierte. In seiner eigentlichen Profession, der Geschichtswissenschaft, galt er als eine der Galionsfiguren der politischen Geschichtsschreibung und zudem als Verfasser eines vielzitierten Buches über die drei großen Richtungen der politischen Rechten. Interessanterweise stellt er darin den Gaullismus in die Tradition des Bonapartismus, einer Strömung innerhalb der französischen Rechten, die gleichermaßen autoritäre wie volksnahe Züge hat. 
Gestützt in erster Linie auf den Privatnachlass Rémonds und das Universitätsarchiv Nanterre, kann Mercier in René Rémond et Nanterre. Les enfantements de 68 den Aufstieg in Amt und Würden im Detail nacherzählen: vom Vizedekan zum Dekan der Fakultät Nanterre, dann zum gewählten Universitätspräsidenten und schließlich zu einem der Vizepräsidenten der Konferenz der Universitätspräsidenten (die Präsidentschaft oblag dem Ministerium). Rémond bekam eine Dienstwohnung, ein Dienstauto und einen Chauffeur gestellt, und wie es scheint, wäre er darüber hinaus auch einem Staatsamt, etwa einem Ministerposten, nicht abgeneigt gewesen. Dass ein Universitätspräsident in die Politik geht, ist auch durchaus nicht ungewöhnlich. Leitete Woodrow Wilson nicht die Princeton Universität, bevor er zum Präsidenten der Vereinigten Staaten gewählt wurde? Doch aus einer politischen Karriere wurde für Rémond am Ende nichts. Dafür lenkte er über Jahrzehnte hinweg die Geschicke von Sciences Po. Bis drei Monate vor seinem Tod blieb er dort Präsident, ehe er 2007 im Alter von 88 Jahren verstarb.

Zur ersten Feuerprobe wurde für Rémond Nanterre. Diese Hochburg der 68erRevolte war ein schwieriges Pflaster für alle Professoren, erst recht für die an der Spitze der Hierarchie. Hier hatte die Bewegung des 22. März mit Daniel Cohn-Bendit ihren Anfang genommen und am Tag ihrer Gründung sogleich das Verwaltungshochhaus gestürmt und den Saal für die Fakultätsratssitzungen, also den Sitz der universitären Macht, besetzt. Hier ereignete sich auch einer der bestpublizierten Zwischenfälle jener Zeit überhaupt. Der Philosoph Paul Ricœur, damals Dekan der Fakultät (mit Rémond als Vizedekan), wurde auf dem Weg zu einer Kaffeepause bespuckt und belästigt, bevor einer der Angreifer ihm den Deckel eines Mülleimers über den Kopf hielt. Vergleichbares passierte übrigens auch in Deutschland, genauer gesagt, in Heidelberg, wo eine Studentin dem dortigen Dekan eine Pickelhaube aufsetzen wollte, "Jahrgang 1910“, wie der Betroffene hinzufügte. Rémond selbst blieb ebenfalls nicht verschont. Eine erste leidvolle Erfahrung machte er bereits am 2. Mai 1968, zu Beginn der Unruhen, als er seinen Vorlesungssaal besetzt vorfand. Studenten hatten dort die Regie übernommen und zeigten, im Rahmen eines selbstorganisierten antiimperialistischen Tages, Filme über die US-Intervention in Vietnam. Ermutigt, ja offenbar gedrängt von seinen Hörern, einem Bericht zufolge über 200, versuchte er, sich dennoch Zutritt zu verschaffen. Aber eine Sitzbank, von den Besetzern als Wurfgeschoss verwendet, traf inn an den Beinen und warf ihn zu Boden. Über ihm flogen sogleich die Fäuste, die Internationale wurde intoniert; der Saal aber blieb weiterhin besetzt.

Dass Rémond der Bewegung zumindest in den ersten Wochen kritisch gegenüberstand, ist unstreitig. Dann aber, wie Mercier berichtet, änderte sich unversehens seine Haltung. Die möglichen Gründe dafür diskutiert er mit großer Umsicht und aus allen erdenklichen Perspektiven. Tatsache bleibt aber, dass Rémond ab Mitte Mai 1968 mit einem Mal Verständnis für die studentischen Forderungen signalisierte und einige Jahre später, in einem Erinnerungsband, der zugleich politischer Essay ist, La Règle et le Consentement, sich selbst sogar als Kind der Bewegung bezeichnet hat. 
Für Mercier ist dies jedenfalls Anlass genug, die Äußerung in den Titel seines Bandes über Nanterre aufzunehmen, allerdings im Plural: „enfantements". Ähnlich verfährt er auch mit dem Titel seines anderen Bandes, dem zu Rémonds Rolle als einer der Wortführer der französischen Universitätspräsidenten: Autonomie, autonomies. René Rémond et la politique universitaire aux lendemains de Mai 1968. Auch hier setzt Mercier bewusst einen Begriff in den Plural: „autonomies“. Dies ist, möchte man sagen, auch ganz im Geiste Rémonds. Schließlich war dessen wichtigstes politisches Credo das des Pluralismus. So wie die 68er-Bewegung, will uns der Autor wohl bedeuten, verschiedenartige Kinder hervorbrachte, darunter eben auch Männer der politischen Mitte und gläubige Katholiken wie Rémond, so ermöglichte die Gesetzgebung Faures unterschiedliche Ausdeutungen universitärer Autonomie. Die Rémonds, folgt man Mercier, war natürlich ebenfalls eine der Mitte. Sie stand zwischen dem Wunsch nicht weniger 68er, eine sehr weitgehende universitäre Selbstverwaltung gegen jede staatliche Einflussnahme durchzusetzen, und der sehr wirtschaftsliberalen Interpretation von Autonomie, die rein marktorientierte Ziele verfolgt. Rémond verkörperte dagegen den Ausgleich: er setzte auf Subsidiarität, auf ein Miteinander auf allen Ebenen, von den Universitäten über die Rektoren bis hinauf zum Ministerium. So übertrug Rémond, wie Mercier bemerkt, ein grundlegendes Prinzip des sozialen Katholizismus auf die Universitätspolitik.

Dass Rémond ein Mann der Mitte war, kam ihm nicht zuletzt auch bei seiner Wahl zum Universitätspräsidenten entgegen. Auf sich vereinigte er so nämlich Stimmen aus dem linken wie dem rechten Lager. Seine Mehrheit umfasste ein breites Spektrum aller reformwilligen Kräfte und reichte von der gemäßigten Linken, den sogenannten Partizipationisten, bis zu ihrem Gegenpart auf der Rechten, von Mercier reformorientierte Liberale genannt; hinzu stießen außerdem noch die Kommunisten. Keine Unterstützung erhielt Rémond dagegen von den sogenannten Unversöhnlichen, also jenen Kräften, die keine Kompromisse eingehen wollten, seien sie nun Teil der Linken oder der Rechten. Insbesondere aber galt dies für die revolutionäre Linke, die jede Beteiligung an einem, wie sie es sah, staatlich kontrollierten, mithin repressiven Regime ablehnte. In letzter Konsequenz schloss das auch die Beteiligung an Universitätswahlen aus.

Einmal gewählt, erwies sich Rémond als pragmatischer, ergebnisorientierter und machtbewusster Präsident. Wie Mercier besonders herausstellt, legte Rémond seine Befugnisse so extensiv aus, dass dies letztlich auf Kosten anderer Gremien und gewählter Vertreter ging. Für Mercier stand dahinter eine gaullistische, also präsidentialistische, Lesart der Gesetzestexte. Dank seiner immensen Autorität erreichte es Rémond jedenfalls, dass unter seiner Leitung nicht nur Tagesordnungen strikt eingehalten, sondern auch einmal getroffene Entscheidungen selbst gegen Widerstand mit fester Hand umgesetzt wurden. So wichtig Rémond ein effizientes Arbeiten aber auch war, so mag man doch bezweifeln, dass er das in gaullistischer Manier tat und dafür bereit war, die Suche nach einem tragbaren Kompromiss zurückzustellen. Denn zugleich lag 
ihm immer auch viel am Dialog und der gemeinsamen Suche nach Lösungen, mit einem Wort, an einer ebenso sachorientierten wie vertrauensvollen Zusammenarbeit. Bestätigung für diese These findet sich unter anderem in Rémonds wissenschaftlichen Arbeiten. Jedenfalls nahm er als Historiker die III. Republik, die ja nun alles andere als präsidentialistisch war, stets gegen ihre Verächter in Schutz, und in seiner Studie über die französische Rechte stufte er darüber hinaus die Gaullisten als Erben des Bonapartismus ein, eine politische Richtung, die nun wahrlich nicht seiner eigenen entsprach. So gesehen wäre es also eine große Überraschung, wenn Rémond seinen Präsidentenposten in einer Art und Weise ausgefüllt hätte, die an die de Gaulles erinnert; zumindest dürfte dies nicht seiner politischen Selbsteinschätzung entsprochen haben. Stattdessen war es wohl eher so, dass er weniger autoritär war als vielmehr ein Mann für die zwar sehr energische, aber stets kollegiale Leitung von Gremien, Kommissionen und nicht zuletzt wissenschaftlichen Kongressen.

In dieses Bild passt schließlich auch, dass Rémond seine Rolle in Nanterre, die Mercier als sehr konstruktiv und erfolgreich beurteilt, mit zwei fehlgeschlagenen Kandidaturen für die Académie des sciences morales et politiques vergolten wurde. Man lehnte inn dort wegen seiner konzilianten Haltung zur 68erBewegung als politisch zu weit links ab. Entschädigt wurde er dann aber 1998, dreißig Jahre nach den Mairevolten, als er schließlich doch noch den grünen Rock überziehen durfte und an die Académie française gewählt wurde.

Insgesamt gesehen beschreibt Mercier das Wirken Rémonds als Administrator und Universitätspolitiker mit großer Sympathie und dabei auch sehr gewissenhaft. Was seinen beiden Bänden jedoch fehlt, ist ein Innehalten und Nachdenken, das vielleicht erlaubt hätte, noch etwas tiefer in die Gedankenwelt Rémonds einzudringen und einen besseren Einblick in sein Weltbild zu erhalten. Pluralismus oder, wie Mercier meist schreibt, politischer Liberalismus sind in der Tat die entscheidenden Begriffe. Aber was bedeuten sie genau für einen Katholiken wie Rémond? Und wie unterscheidet sich sein Liberalismus von dem eines Nicht-Katholiken? In welchem Zusammenhang stehen außerdem der liberale Katholizismus des 19. Jahrhundert oder, genauer, die Ideen eines Lamennais (über den Rémond seine ersten Arbeiten schrieb) mit seinem Wirken in Nanterre? Und nicht zuletzt: gibt es Parallelen zwischen seiner Auffassung vom Verhältnis von Kirche und Staat und dem von Universität und Staat?

Fairerweise muss man sagen, dass Mercier ja ausdrücklich keine Biographie schreiben wollte. Doch auch in der Biographie, die er vor kurzem dann doch vorgelegt hat und die unter dem Titel René Rémond. Une traversée du XXe siècle erschienen ist, tut er sich mit derartigen Fragen schwer. Dies liegt nicht zuletzt daran, dass sie vor allem jene frühen Jahre der intellektuellen Entwicklung Rémonds betreffen, die sein Nachlass bedauerlicherweise nicht abdeckt. Dieser enthält lediglich Dokumente aus der Zeit seit 1968, also sozusagen seit der Übernahme einer nach und nach immer bedeutenderen öffentlichen Rolle, und so findet sich darin auch nichts zu seinem Engagement in der katholischen Studentenjugend oder seinem Wirken im Zentrum der katholischen Intellektuellen. Das ist natürlich schade. Doch dessen ungeachtet sind die Bände 
Merciers nicht nur eine beeindruckende Forschungsleistung, sondern darüber hinaus auch sehr angenehm zu lesen.

Im Gegensatz zu Mercier zeigt Christelle Dormoy-Rajramanan keinerlei Interesse an einer rein biographischen Arbeit. Ihre Welt ist vielmehr die der sozialen Gruppen, der Eliten etwa oder der Staatsbeamten. So beklagt sie in einer Rezension denn auch Merciers "monobiographischen" Blick, der ihn dazu verleite, die Bedeutung Rémonds in der Universitätspolitik konstant zu überschätzen. Mit diesem Urteil ist sie keineswegs allein. Es ist die altbekannte Kritik einer soziologisch orientierten Geschichtswissenschaft an allen historischen Biographien. Obwohl Dormoy-Rajramanan ihre Studie im Fach Politikwissenschaft eingereicht hat, stammen viele ihrer Thesen aus einem Wissenschaftszweig, wo Sozialgeschichte und die Soziologie eng miteinander verwachsen sind. Es geht ihr also nicht um sich schnell verflüchtigende politische Mehrheiten, sondern um langlebige soziale Trends und Entwicklungen; sie stellt auch nicht verschiedene Deutungen gegenüber und wägt behutsam ab, so wie Mercier es tut, statt dessen leitet sie eines aus dem anderen her und sucht nach einem systematischen Zusammenhang oder, besser gesagt, einem System.

Dieses System entstand ihrer Meinung nach schon 1944 oder, noch früher, in der späten III. Republik. Darum setzt Dormoy-Rajramanans Analyse auch nicht im Sommer und Herbst 1968 ein, als Vincennes erdacht und erbaut wurde, sondern Jahrzehnte davor. Ihre Absicht ist es, die restriktiven sozialen Bedingungen des französischen Bildungssystems herauszuarbeiten und auf diese Weise die Umstände der Gründung von Vincennes zu beleuchten. So entstand eine Soziogenese, eine sozialhistorische wie soziologische Untersuchung über die Entstehungsgeschichte der Pilotuniversität Vincennes: Sociogenèse d'une invention institutionnelle. Le Centre universitaire expérimental de Vincennes. Während die Systemgeschichte, unterfüttert mit einer beeindruckenden Menge empirischer Daten, jedoch auf mehreren hundert Seiten ausgebreitet wird, umfasst der Teil zu Vincennes lediglich 150 Seiten und setzt sich zudem weitgehend aus bereits veröffentlichten Artikeln zusammen. Dass sich dessen Lektüre dennoch lohnt, liegt an der großen Zahl neuer Quellenfunde, darunter insbesondere solche aus Archiven gaullistischer Verantwortlicher. Sie dokumentieren hautnah die Reaktionen auf die Entwicklung in Vincennes, die zwischen heller Aufregung und handfestem Entsetzen schwanken.

Was also ist nun die große, allumfassende These der Arbeit DormoyRajramanans? Und wie fügt sich Vincennes darin ein? Ausgangspunkt ist die Annahme, dass das entscheidende Merkmal des Schul- und Universitätssystems seit der III. Republik nicht die Demokratisierung ist, also die Öffnung hin zu allen Bevölkerungsschichten, sondern der unbedingte Wille, es nach marktwirtschaftlichen Gesichtspunkten neu zu ordnen. Folgt man DormoyRajramanan, spielen die Interessen von Industrie und Wirtschaft somit eine herausgehobene Rolle und werden in Politik und Verwaltung unterstützt. Dass 
infolge der Demokratisierung des Bildungssystems die Zahl der Studenten rapide zunimmt, werde - so die Autorin - von allen Seiten durchaus willkommen geheißen. Doch um sich aller Nachwuchssorgen zu entledigen, bedarf es aus Sicht der Wirtschaft, wie Dormoy-Rajramanan weiter ausführt, zudem einer gesonderten Unterstützung der naturwissenschaftlichen Fächer; denn für die Mehrheit der Studenten sind diese im Vergleich zu Geistes-, Sprach- und Sozialwissenschaften nicht attraktiv genug. Demzufolge ist das erklärte Ziel also, die Universitäten dazu zu bringen, nur für den tatsächlichen Bedarf auf dem Arbeitsmarkt auszubilden. Diese Forderung ist der Kern einer ganzen Reihe von Reformbestrebungen, die Dormoy-Rajramanan eingehend analysiert. Darunter sind bekannte Wegmarken wie der Langevin-Wallon Plan von 1947, das von Mendès France initiierte Kolloquium in Caen von 1966 und der landesweite Ausbau rein technisch-wirtschaftlicher Bildungsstätten in den 1960er Jahren unter de Gaulle. Zu den Eigentümlichkeiten des Systems gehört laut DormoyRajramanan nun, dass die Revolte der Studenten im Mai 1968 nicht nur maßgeblich durch den jahrzehntelangen Reformdruck ausgelöst wurde, sondern dass die Krise mit Hilfe ebenjener Reformen auch wieder bewältigt werden sollte, genauer gesagt: durch den abermaligen Rückgriff auf die Standardmittel der Ökonomisierung und Professionalisierung nach dem Vorbild amerikanischer Universitäten. Ein nicht unwesentlicher Eckpunkt dieser Maßnahmen sind demnach die beiden neu gegründeten Pariser Versuchsuniversitäten: Dauphine und Vincennes. Sie sollten als Pilotprojekte zeigen, wie eine Hochschule, organisiert nach modernen ökonomischen Prinzipien, in der Praxis funktionieren kann.

Das also ist, zusammengefasst in gebotener Kürze, das System, das im Zentrum der Arbeit von Dormoy-Rajramanan steht. Der Vollständigkeit halber müssen darüber hinaus aber noch die technokratischen, reformorientierten Eliten erwähnt werden, die Beamten, Wissenschaftler, Politiker und Unternehmer, die die eigentlichen Akteure sind. Sie bestimmen gemeinschaftlich, als soziale Gruppe und Träger der dominanten Ideologie, die Rahmenbedingungen der französischen Bildungspolitik, und das parteiübergreifend, fern aller ideologischer Kontroversen und selbst über den Wandel der Republiken hinweg. Die Gründe für die Entstehung von Vincennes sind infolgedessen nicht im politischen Willen de Gaulles oder im Realitätssinn Faures zu suchen und auch nur in zweiter Linie im erfolgreichen Kampf der 68er-Bewegung; möglich machte Vincennes vielmehr der unablässige Reformdruck, der schon vor 1968 Bestand hatte und genauso nach 1968 Bestand haben wird.

So theoretisch und abstrakt diese weitausgreifende Theorie auch klingen mag, so offenbart sich in ihr gleichwohl die persönliche Überzeugung der Autorin. Jedenfalls ist nur schwer zu übersehen, dass Dormoy-Rajramanan in ihren Arbeiten mit allem Nachdruck davor warnen will, die Universitätsausbildung nur noch als Vorbereitung auf den Beruf, nicht aber als Erziehung zum kritischen Staatsbürger zu verstehen. Oder bildlicher formuliert, sie ist der Auffassung, dass sich die Verantwortlichen zu lange schon auf ihren Tanz um das eine goldene Kalb konzentriert haben: den Markt. 
Angesichts der im Grunde doch recht kurzen Geschichte von Vincennes ist der theoretische Überbau der Studie Dormoy-Rajramanans allerdings ein außerordentlich lang geratener Prolog. Dabei hätte man, statt mit den fernen Anfängen, auch genauso gut mit dem Ende beginnen können. Eindrücklich sind etwa die Momente, in denen in Vincennes, l'université perdue, einer ARTEDokumentation, die Zeitzeugen auf einem verlassen dastehenden Stuhl inmitten einer Waldlichtung interviewt werden. Es ist der Ort, an dem die Versuchsuniversität einst ihren Sitz hatte. Nostalgie und Melancholie lassen sich dabei mit Händen greifen, aber auch Wut und ein eigentümliches Gefühl der Ohnmacht. Vincennes jedenfalls, eines der Symbole der 68er-Bewegung, ist inzwischen Geschichte. Nach nur knapp 12 Jahren wurden auf Geheiß der französischen Regierung alle Universitätsgebäude abgerissen und der Lehrkörper samt Studenten und Angestellten nach Saint-Denis verpflanzt, einem Arbeiterviertel, in dem die Kommunistische Partei das Sagen hatte. Damit wurde Vincennes endgültig, wie Dormoy-Rajramanan es ausdrückt, zu „einer Art Martyrium für die akademische Linke".

Noch mehr als das Bild von der leeren Waldlichtung ist allerdings ein anderes im öffentlichen Gedächtnis haften geblieben: das des Philosophen Michel Foucault, der am 23. Januar 1969 an der ersten Besetzung des Campus durch die Studenten mitwirkte. Geplant waren die Proteste als Tag der studentischen Mobilisierung, mit der Vorführung eines Films über die Ereignisse im vergangenen Mai und Forderungen nach zusätzlichen Stipendiengeldern und Straffreiheit für einzelne Studenten. Dann aber mündeten sie in die nächtliche Besetzung des Campus, woraufhin die Polizei das Gelände gewaltsam räumte. Folgt man einem Augenzeugenbericht, reihte sich Foucault nicht nur solidarisch in die Reihen der rebellierenden Studenten ein, sondern hatte zudem die Idee, einen Feuerlöscher über die anstürmenden Bereitschaftspolizisten zu entleeren; diese wiederum setzten Tränengas ein. Die Bilanz der einen Nacht war niederschmetternd. Es bot sich ein Bild der Verwüstung: die fabrikneuen Designer-Büromöbel, zu Barrikaden aufgetürmt, waren zertrümmert; die Zimmerdecken, in denen Überwachungsmikrofone der Polizei vermutet wurden, aufgerissen; und Foucault, zusammen mit einigen Studenten, abgeführt in eine Zelle der Pariser Polizeipräfektur. Neben dem erheblichen materiellen Schaden erlitt Vincennes überdies einen nicht wieder gut zu machenden Imageschaden.

Dabei hatte die Universität am Pariser Stadtrand nicht nur des Nachts einiges zu bieten. Wichtige Neuerungen, ermöglicht durch die großen Freiheiten, die ihr als Modellhochschule zugestanden wurden, gab es jedenfalls reichlich. So wurden die Mandarine, wie die mächtigen Universitätsprofessoren verächtlich genannt wurden, vom Podest gestoßen. Anstelle der üblichen wenigen Vorlesungen mit vielen hundert Hörern sollten sie, zusammen mit ihren wissenschaftlichen Mitarbeitern, viele kleinere Kurse und Seminare abhalten. Das erklärte Ziel war, auf diese Weise nicht nur die physische Distanz zwischen Studenten und Lehrkörper zu verringern, sondern auch den persönlichen Kontakt einfacher und direkter zu gestalten. Außerdem schaffte man das 
Jahresexamen ab, das für viele Studenten eine echte Belastung war, und führte an dessen Stelle das amerikanische System der Kreditpunkte ein, in ihrer französischen Fassung Unité de valeurs genannt. Schließlich wurden in Vincennes auch Studenten ohne Abitur zum Studium zugelassen, wobei einer von ihnen, der Busfahrer Philippe Mioche, später sogar selbst Universitätsprofessor werden sollte. Der Lehrkörper wiederum nutzte die Freiheiten, die er hatte, um die Fachbereiche von Grund auf umzugestalten. So wurden allerorten neue Disziplinen erschaffen und alte Fächer zu neuen kombiniert. Foucault etwa versuchte sich für seinen Teil an der Verschmelzung von Biologie und Philosophie.

Daneben bestimmte der politische Kampf das Leben auf dem Campus. Eine Vielzahl von Vollversammlungen fanden statt, und auch die Studentenproteste wurden unvermindert fortgesetzt. Aufmerksamkeit über Campusgrenzen hinweg brachte Vincennes insbesondere ein Zwischenfall am 18. Juni 1969. Es war der Tag, an dem die ersten Universitätswahlen stattfinden sollten. Aber einige Studenten machten unmissverständlich klar, was sie von diesen Wahlen und von Wahlen überhaupt hielten. Die bereitgestellten Wahlurnen wurden jedenfalls umgehend entsorgt und landeten im Wasserbecken des Campus. Dabei war dies wohl nur ein weiteres untrügliches Zeichen dafür, dass in der Studentenschaft von Vincennes die Anti-Partizipationisten den Ton angaben und auch zahlenmäßig stärker waren als in Nanterre.

Doch anders als die damaligen Regierungspolitiker macht Dormoy-Rajramanan in ihrer Studie nicht die starke Politisierung für die sich über Jahre hinziehenden Probleme in Vincennes verantwortlich. Vielmehr glaubt sie, dass die Regierung die Universität am langen Arm verhungern ließ. Wie aber soll eine junge Pflanze gedeihen, wenn ihr Licht und Wasser fehlt? Obendrein, so führt sie aus, wurden die Mittel mit voller Absicht gekürzt. Ein Vergleich mit Dauphine, der anderen Pilotuniversität im Pariser Raum, zeige sehr deutlich, wie mit zweierlei Maß gemessen wurde. So habe die eine Hochschule die dringend benötigten Gelder ohne Schwierigkeiten erhalten, während man sie der anderen immer wieder vorenthalten habe. Als Erklärung für die ungleiche Behandlung der beiden Geschwisteruniversitäten nennt Dormoy-Rajramanan zwei Gründe: zum einen den Umstand, dass das Ministerium die Wirtschaftswissenschaften, die in Dauphine unterrichtet wurden, als förderungswürdiger erachtete als die Geistes- und Sozialwissenschaften, die den Schwerpunkt in Vincennes bildeten, und zum anderen die unterschiedliche politische Situation vor Ort, mit zahmen Studenten in Dauphine, sehr rebellischen hingegen in Vincennes. Kein Wunder also, dass das Ministerium, wie Dormoy-Rajramanan meint, unter den beiden Versuchsuniversitäten nur ein Lieblingskind hatte: Dauphine. Darüber hinaus greift die Autorin aber noch ein Gerücht auf, das in jener Zeit kursierte und das ihr als weiterer Beweis für den Paria-Status von Vincennes gilt: die Vermutung nämlich, dass Vincennes und seine linksradikale Professoren- und Studentenschaft genau so aus dem Pariser Zentrum in den Wald von Vincennes ausgelagert wurden, wie man einen Abszess vom gesunden Gewebe abkapselt, in der Hoffnung, dadurch den Patienten als Ganzes retten zu können. 
Durch diesen Vergleich aus der Heilkunde verliert allerdings der zentrale Erklärungsansatz ihrer Arbeit, der ja allein um die Frage der Wirtschaftlichkeit der Universitäten kreist, seine innere Schlüssigkeit. Denn nun kehren all jene politischen Fragen durch die Hintertür zurück, denen Dormoy-Rajramanan zuvor mit Bedacht ausgewichen ist. In ihrer Theorie hat die ungeheure politische Vielfalt Frankreichs ja im Grunde keine Rolle gespielt, nicht die in Regierung, Parlament und Öffentlichkeit und schon gar nicht die in der technokratischen Elite, genauso wenig im übrigen wie die vielgestaltigen politisch-ideologischen Bezüge, die in der 68er-Bewegung zu beobachten sind. Nun aber ist das Politische wieder da, mit Vincennes in der Opferrolle. Dabei muss man sich jedoch fragen, ob die beiden Argumentationsstränge, das politische Ziel der Marginalisierung linksradikaler Studenten und Professoren einerseits und das marktorientierte System andererseits, tatsächlich so ohne weiteres miteinander zu vereinbaren sind. Zumal wenn in dem einen alles auf einen politischen Kampf zwischen Links und Rechts hinausläuft, in dem anderen jedoch ein konstanter, überparteilicher Konsens für eine Ökonomisierung des Universitätssystems herrschen soll.

Die entscheidende Schwachstelle der Studie Dormoy-Rajramanan ist aber die Einbindung der Entstehungsgeschichte von Vincennes in den theoretischen Überbau. Für die These, dass die Pilotuniversität zumindest zu Anfang maßgeblich nach marktwirtschaftlichen Prinzipien konzipiert wurde, fehlt es jedenfalls an überzeugenden Belegen. Es reicht eben nicht aus, nur darauf zu verweisen, dass in Vincennes vereinzelte Studiengänge eingerichtet wurden, die direkt auf Berufe vorbereiten, wie den Journalismus, oder dass der Universitätsrat auch für Persönlichkeiten der außeruniversitären Welt, sprich: der Welt der Firmen und des Staates, geöffnet werden sollte und dass schließlich die durchgehende Benotung, eingeführt als Ersatz für das Jahresexamens, auch als versteckte Selektion gewertet werden könne. Denn demgegenüber steht das Zugeständnis sehr weitgehender wissenschaftlicher, pädagogischer und institutioneller Freiheiten, speziell auch die eigenverantwortliche Kooptation von Professoren und wissenschaftlichen Mitarbeiter. Hätte man nun ausschließlich im Interesse von Industrie und Wirtschaft gehandelt, wäre das Herzstück der Reform nicht gerade die grundlegende Erneuerung der Lehre, des Lehrplans und des Fächerkanons. Man hätte indessen wohl sehr viel mehr Wert gelegt auf die Güte und Verlässlichkeit der Abschlüsse so wie auch auf die Berufstauglichkeit der Ausbildung, und das nicht nur in den Wirtschaftswissenschaften, sondern auch in den Geisteswissenschaften. Zudem: wie hätte man eine derartige Professionalisierung des Studiums angesichts der mannigfaltigen Freiheiten, die der Pilotuniversität Vincennes zugestanden wurden, staatlicherseits überhaupt garantieren sollen? Keine Frage, es liegen Welten zwischen der These, Vincennes sei ursprünglich als universitäre Spielwiese gedacht gewesen, und der Dormoy-Rajramanans, Vincennes sei zumindest zu Beginn den Bedürfnissen der Wirtschaft entsprechend entworfen worden. Weitaus plausibler freilich ist die erste dieser beiden Hypothesen. 
Die Zeit der Freiheit sollte dann aber recht bald enden. So entschied Olivier Guichard, Erziehungsminister und Nachfolger Edgar Faures, bereits im Januar 1970 den Abschlüssen an der philosophischen Abteilung von Vincennes die staatliche Anerkennung zu verweigern. Nun war es also am Staat, verlorenes Terrain zurückzugewinnen. Mehr staatliche Kontrolle über die Universitäten: so lässt sich die Tendenz der Politik in den 1970er Jahre vielleicht am besten beschreiben. Einer der Hebel dafür war der Umstand, dass ein Universitätsdiplom die Zustimmung des Ministeriums bedurfte, um als „nationales Diplom“ anerkannt zu werden, der andere war das Budget, also die Drohung, Gelder zu kürzen, für den Fall, dass die Weisungen des Ministeriums nicht beachtet wurden. Mittels dieser beiden Hebel, wie es vor allem Arnaud Desvignes in seinen Arbeiten aufzeigt, wurde die von Faure gewährte Autonomie nicht nur in Frage gestellt, sondern mehr und mehr zurückgedrängt. Die allgemeine Entwicklung betraf auch den Universitätspräsidenten René Rémond, für den Charles Mercier zufolge - vor allem das Jahr 1976 zur eigentlichen Zäsur wurde. Es war das Jahr, in dem seine politische Gegenspielerin, Alice Saunier-Seïté, das Ressort der Universitäten übernahm und die Regierung mit Nachdruck zu einer rechten Universitätspolitik zurückführte. Für ihre Verdienste wurde die Ministerin, anders als Rémond, dann im Übrigen auch mit der Wahl an die Académie des sciences morales et politiques belohnt.

Welch heißes Eisen die Frage der universitären Autonomie auch heute noch ist, zeigt eine lebhafte Auseinandersetzung anlässlich des hier besprochenen Kolloquiums über die Universitätsgeschichte in der Île-de-France. Dem Historiker Antoine Prost, der die staatliche Einflussnahme vehement kritisiert, steht Jean-Yves Mérindol gegenüber, ein Praktiker und selbst Universitätspräsident, der die großen Freiräume der Universitäten in den 1970er Jahren rühmt. Wieviel Autonomie allerdings der französische Staat seinen Universitäten wirklich zugesteht, scheint stets neu verhandelt zu werden. Das System ist also im Fluss. Dennoch ist es immer noch weit entfernt vom Humboldtschen Ideal freier Universitäten, mit einem Staat, der allenfalls als Schutzmacht für die wissenschaftliche Freiheit in Erscheinung tritt. Doch spätestens seit der Revolution Edgar Faures ist es auch nicht mehr das Modell Napoleons, in dem die Universitäten einer rigiden staatlichen Kontrolle unterworfen sind.

So kunterbunt und unübersichtlich, wie sich die französische Universitätslandschaft heutzutage darstellt, so ist dann auch die Forschung, die sich mit ihr beschäftigt. Die Bände, die hier vorgestellt wurden, zeigen jedenfalls ein wahres Kaleidoskop verschiedenartiger Ansätze, Thesen und Meinungen. Dabei sollte man aber nicht vergessen, wer dies erst möglich gemacht hat: die Archivarinnen und Archivare. 
